\title{
Surgery for incarcerated hernia: short-term outcome with or without mesh
}

\author{
A. Venara • M. Hubner • P. Le Naoures • J. F. Hamel • \\ A. Hamy $\cdot$ N. Demartines
}

Received: 2 December 2013 / Accepted: 21 April 2014 / Published online: 1 May 2014

(C) Springer-Verlag Berlin Heidelberg 2014

\begin{abstract}
Background Incarcerated hernias represent about 5-15\% of all operated hernias. Tension-free mesh is the preferred technique for elective surgery due to low recurrence rates. There is however currently no consensus on the use of mesh for the treatment of incarcerated hernias, especially in case of bowel resection. Aim The aims of this study were (i) to report our current practice for the treatment of incarcerated hernias, (ii) to identify risk factors for postoperative complications, and (iii) to assess the safety of mesh placement in potentially infected surgical fields. Methods This retrospective study included 166 consecutive patients who underwent emergency surgery for incarcerated hernia between January 2007 and January 2012 in two university hospitals. Demographics, surgical details, and short-term outcome were collected. Univariate analysis was employed to identify risk factors for overall, infectious, and major complications. Results Eighty-four patients $(50.6 \%$ ) presented inguinal hernias, 43 femoral (25.9\%), 37 umbilical hernias ( $22.3 \%$ ), and 2 mixed hernias (1.2\%), respectively. Mesh was placed in 64 patients (38.5\%), including 5 patients with concomitant bowel resection. Overall morbidity occurred in 56 patients
\end{abstract}

A. Venara and M. Hubner have shared first authorship.

A. Venara $(\varangle) \cdot$ P. Le Naoures $\cdot$ A. Hamy

Visceral and Endocrine Surgery Department, University Hospital of Angers, 4 rue Larrey, 49933 Angers Cedex 9, France

e-mail: auvenara@yahoo.fr

A. Venara $\cdot$ M. Hubner $\cdot$ N. Demartines

Department of Visceral Surgery, University Hospital CHUV, Rue du

Bugnon 46, 1011 Lausanne, Switzerland

P. Le Naoures $\cdot$ J. F. Hamel $\cdot$ A. Hamy

L'UNAM, University of Angers, Angers, France

J. F. Hamel

Department of Methodology and Biostatistics, University Hospital of Angers, 4 rue Larrey, 49933 Angers Cedex 9, France
(32.7\%), and 8 patients $(4.8 \%)$ developed surgical site infections (SSI). Univariate risk factors for overall complications were ASA grade $3 / 4(P=0.03)$, diabetes $(P=0.05)$, cardiopathy $(P=0.001)$, aspirin use $(P=0.023)$, and bowel resection $(P=0.001)$ which was also the only identified risk factor for SSI $(P=0.03)$. In multivariate analysis, only bowel incarceration was associated with a higher rate of major morbidity $(\mathrm{OR}=14.04 ; P=0.01)$.

Conclusion Morbidity after surgery for incarcerated hernia remains high and depends on comorbidities and surgical presentation. The use of mesh could become current practice even in case of bowel resection.

Keywords Hernia $\cdot$ Incarceration $\cdot$ Mesh $\cdot$ Surgical site infection $\cdot$ Bowel resection

\section{Introduction}

Incarcerated hernias represent between 5 and $15 \%$ of groin hernia repairs [1-4] and about $10 \%$ of operated umbilical hernias [5]. In elective hernia surgery, tension-free mesh repair (open or endoscopically) has been proven to be more effective than suture reconstruction (odds ratio $=0.43$ ) in terms of longterm recurrence [6-10]. Wound infection rates vary between 1 and $7 \%$ in both mesh and no-mesh repair [6].

Recommendations differ for the treatment of incarcerated hernias which are typically performed as emergency procedures. The use of prosthetic material is generally avoided, especially in a septic environment such as in case of concomitant bowel resection. Recent reports challenged these preconceptions. The first study comparing mesh and no-mesh in strangulated groin hernia repair was published in 2008 and reported favorable outcomes for the mesh group [11]. These initial data were confirmed recently showing similar infection rates but reduced recurrence rates in the long-term follow-up 
in the mesh group [11-14]. These results have been reproduced even in the context of concomitant bowel resection $[15,16]$. So far, no consensus has been reached on mesh placement for the treatment of incarcerated hernias.

The aims of our study were (i) to report our current practice for incarcerated hernia repair, (ii) to identify risk factors for postoperative complications, and (iii) to assess the safety of mesh placement during contaminated surgery (class 2, 3) [17-19].

\section{Methods}

This retrospective study included all consecutive patients $>18$ years who underwent emergency repair for incarcerated hernia between January 2007 and January 2012 in two tertiary referral centers, the University Hospital of Lausanne, Switzerland, and of Angers, France. This study obtained approval by the ethical committees of both institutions.

Only true emergency cases were considered. Emergency surgery was defined as any intervention performed within $6 \mathrm{~h}$ after hospital admission. Inguinal, femoral, and umbilical hernias were included; exclusion criteria were recurrent or incisional hernias, elective surgeries, and patients presenting with ascites or frank peritonitis requiring laparotomy. Incarceration was defined based on clinical grounds as nonreducible, painful hernia associated with nonimpulsiveness during Valsalva maneuver [20].

The surgeon chose the surgical access according to his habits and his knowledge.

Data were retrospectively retrieved from the medical files and entered anonymously into an electronic database. Items to collect and outcome measures were defined a priori. Collected data included (i) demographic information such as age, gender, American Society of Anesthesiologists (ASA) grade, body mass index (BMI), usual medication, and comorbidities. Obesity was defined by a BMI $>30 \mathrm{~kg} / \mathrm{m}^{2}$ [21].

(ii) Surgical details were type of hernia (femoral, inguinal, umbilical), aspect of the bowel (normal, congestive, necrotic), aspect of eventual liquid in the hernia sac (clear or turbid), intestinal resection, prosthetic material, and the technique of repair. (iii) Clinical outcome measures were length of stay (admission-discharge) and overall, infectious, and major complications. Complications were classified according to their severity using a validated 5-grade scale [22]. Major complications were defined as grade $>$ II. Surgical site infection (SSI) was defined according to the CDC as any wound infection occurring within 30 days after operation and was classified as superficial incisional SSI (skin and subcutaneous tissue) or deep incisional SSI (deep and soft tissue) [23].

Length of follow-up was set at 45 days and corresponded with the usual date of routine postoperative control.

Following descriptive analysis, we compared patients with complications to patients without complications. Further analyses were performed with regard to the occurrence of major complications and surgical site infections, respectively.

Data are expressed as mean $\pm \mathrm{SD}$ or median (range) as appropriate. Chi-2 square was used for the comparison of categorical variables. Student's $t$ test and Mann-Whitney $U$ test were employed to compare normal and nonnormal continuous variables. $P<0.05$ was considered statistically significant. Data analysis was performed with the Statistical Package for the Social Science version 15 Software (SPSS, Chicago, IL).

Multivariate logistic regressions were performed for studying the factors associated with complications. The model selection was performed using the Akaike criterion. Femoral hernia was considered as the reference for comparing the three kinds of hernias.

\section{Results}

During the 5-year study period, 166 unselected patients underwent emergency hernia surgery according to the strict inclusion criteria in the two participating hospitals. Demographics are displayed in Table 1. Seventy-six patients

Table 1 Demographics of patients with emergency repair of incarcerated hernia

\begin{tabular}{ll}
\hline & Number of patients (\%) \\
\hline Age (years) & $74(18-109)$ \\
Gender & \\
Male & $97(58.4)$ \\
Female & $69(41.6)$ \\
ASA grade & \\
I/II & $55(30.1)$ \\
III/IV & $23(19.9)$ \\
Unknown & $78(50)$ \\
Comorbidities & \\
Hypertension & $79(47.6)$ \\
Diabetes & $25(15.1)$ \\
Bronchial asthma & $23(13.9)$ \\
Cardiopathy & $52(31.3)$ \\
Obesity & $26(15.7)$ \\
Treatment & \\
Antivitamin K & $17(10.2)$ \\
Aspirin & $31(18.7)$ \\
Immunosuppression & $8(4.8)$ \\
Type of hernia & \\
Groin hernia & $129(77.7)$ \\
Inguinal & $84(65.1)$ \\
Femoral & $43(33.3)$ \\
Femoral + inguinal (mixed) & $2(1.6)$ \\
Umbilical hernia & $37(22.3)$ \\
\hline & \\
\hline & \\
\hline &
\end{tabular}


(45.8\%) were operated in Lausanne (Switzerland), and 90 patients (54.2\%) in Angers (France).

Eighty-four patients $(50.6 \%)$ presented with incarcerated inguinal, 43 femoral (25.9\%), 2 mixed (1.2\%), and 37 umbilical hernias $(22.3 \%)$, respectively. Bowel resection was required in 25 patients (15.1\%). Mesh was placed in 64 patients $(38.5 \%)$ including four patients with concomitant bowel resection $(6.2 \%)$. Surgical details are given in Table 2.

Short-term follow-up was available for $100 \%$ of the patients. Overall morbidity occurred in 56 patients $(33.7 \%)$. Some patients had one or more complications. Class I complications (urinary retention, hematoma, seroma, ileus, and other nonspecific complications) occurred in 18 cases $(32.1 \%)$, class II complications (urinary retention or infection, superficial wound infection, hematoma, pneumopathy, and nonspecific complications) in 16 patients $(28.6 \%)$, class III complications (superficial and deep SSI, recurrence and ileus) in 13 patients $(23.2 \%)$, and class IV complications (multivisceral dysfunction on anastomotic fistula and sepsis from undetermined origin) occurred in 2 patients $(3.6 \%)$ (Table 3). In short-term follow-up, one and two patients developed recurrence after suture and mesh repair, respectively.

Nonspecific complications (not directly due to surgery) were in particular the following: acute coronaropathy, mesenteric ischemia, sepsis, disorientation, and acute hepatic failure.

Table 2 Surgical details of emergency repair of incarcerated hernia

\begin{tabular}{|c|c|c|c|}
\hline & \multicolumn{3}{|l|}{ Inguinal hernia } \\
\hline & Mesh, $n=40(47.6 \%)$ & \multicolumn{2}{|c|}{$\begin{array}{l}\text { No mesh, } n=44 \\
\quad(52.4 \%)\end{array}$} \\
\hline & Resection No resection & resection & No resection \\
\hline \multirow[t]{4}{*}{ Number of patients } & 38 & 5 & 39 \\
\hline & \multicolumn{3}{|l|}{ Umbilical hernia } \\
\hline & Mesh, $n=9(24.3 \%)$ & \multicolumn{2}{|c|}{$\begin{array}{l}\text { No mesh, } n=28 \\
\quad(75.7 \%)\end{array}$} \\
\hline & Resection No resection & Resection & No resection \\
\hline \multirow[t]{4}{*}{ Number of patients } & 2 & 5 & 23 \\
\hline & \multicolumn{3}{|l|}{ Femoral hernia } \\
\hline & Mesh, $n=15(34.9 \%)$ & \multicolumn{2}{|c|}{$\begin{array}{l}\text { No mesh, } n=28 \\
\quad(65.1 \%)\end{array}$} \\
\hline & Resection No resection & Resection & No resection \\
\hline \multirow[t]{4}{*}{ Number of patients } & 15 & 10 & 18 \\
\hline & \multicolumn{3}{|l|}{ Femoral and inguinal hernia } \\
\hline & Mesh & \multicolumn{2}{|c|}{ No mesh, $n=2(100 \%)$} \\
\hline & Resection No resection & Resection & No resection \\
\hline \multirow[t]{4}{*}{ Number of patients } & 0 & 1 & 1 \\
\hline & \multicolumn{3}{|l|}{ Incarcerated hernia: total } \\
\hline & Mesh, $n=64(38.6 \%)$ & \multicolumn{2}{|c|}{$\begin{array}{l}\text { No mesh, } n=102 \\
\quad(61.4 \%)\end{array}$} \\
\hline & Resection No resection & Resection & No resection \\
\hline Number of patients & 60 & 21 & 81 \\
\hline
\end{tabular}

Table 3 Postoperative complications-overview

\begin{tabular}{lc}
\hline & Number of patients (\%) \\
\hline Overall morbidity & $56(33.7)$ \\
Medical complications & $47(28.3)$ \\
Urinary infection & $4(2.4)$ \\
Urinary retention & $12(7.2)$ \\
Pneumopathy & $4(2.4)$ \\
Other & $27(16.3)$ \\
Surgical complications & $30(1.8)$ \\
Recurrence within 30 days & $4(2.4)$ \\
Hematoma/seroma & $12(7.2)$ \\
Superficial incisional SSI & $5(3)$ \\
Deep incisional SSI & $3(1.8)$ \\
Anastomotic fistula & $1(0.6)$ \\
Ileus & $3(1.8)$ \\
Mortality & $7(4.2)$ \\
\hline
\end{tabular}

Mortality (class V) occurred in seven patients (4.2\%) (mortality was due to heart failure in three patients and pulmonary embolism, acute hepatic failure, acute hemorrhage, and mesenteric ischemia in one patient each).

On univariate analysis, patients with bowel resection, aspirin, cardiopathy diabetes, and ASA grade $=3-4$ had a significant higher risk for overall complications $(P<0.001, P=$ 0.02, $P=0.001, P=0.04$, and $P=0.02$ ) (Table 4).

Resection, small bowel incarceration, and femoral hernias were univariate risk factors for major complications $(P=$ 0.003, $P=0.04$, and $P=0.04$ ) (Table 5).

Eight patients develop SSI (4.8\%). Five of them were superficial infection. The other three patients presented mesh infections. Among these eight patients, two patients had a bowel resection (one with prosthetic infection and one with superficial wound infection). All patients with infections were managed without removal of the mesh. The only significant risk factor for surgical site infection (SSI) was the incarceration of colon $(P=0.03)$ (Table 6).

In multivariate analysis, no interaction was found between the use of prosthesis and the rate of overall complications or the rate of major complications (Tables 7 and 8). Among the criterion studied, only ASA score and incarceration of bowel (small bowel or colon) were associated with a higher rate of complications, whereas only incarceration of bowel was associated with a higher rate of major complications.

\section{Discussion}

Incarcerated hernias entail high complication rates which depend on comorbidities and the severity of the intraoperative presentation. However, postoperative SSI remains low, and 
Table 4 Univariate risk factors for postoperative complications

Entries in bold inside the table are stastistically significant results

\begin{tabular}{lllll}
\hline & $\begin{array}{l}\text { No complications, } \\
\%(n=110)\end{array}$ & $\begin{array}{l}\text { Complications, } \\
\%(n=56)\end{array}$ & Total. \% $(n=166)$ & $P$ value \\
\hline Female & $43.6(N=48)$ & $37.5(N=21)$ & $41.6(N=69)$ & 0.45 \\
Cardiopathy & $\mathbf{2 2 . 7 ( n = 2 5 )}$ & $\mathbf{4 8 . 2 ( n = 2 7 )}$ & $\mathbf{3 1 . 3}(\boldsymbol{n}=\mathbf{5 2})$ & $\mathbf{0 . 0 0 1}$ \\
BPCO & $12.7(n=14)$ & $16.1(n=9)$ & $13.9(n=23)$ & 0.7 \\
Diabetes & $\mathbf{1 0 . 9}(\mathbf{n = 1 2})$ & $\mathbf{2 3 . 2}(\boldsymbol{n}=\mathbf{1 3})$ & $\mathbf{1 5 . 1}(\boldsymbol{n}=\mathbf{2 5})$ & $\mathbf{0 . 0 4}$ \\
Obesity & $15.5(n=17)$ & $16.1(n=9)$ & $15.1(n=25)$ & 0.91 \\
Aspirin & $\mathbf{1 3 . 6 ( n = 1 5 )}$ & $\mathbf{2 8 . 6}(\boldsymbol{n}=\mathbf{1 6})$ & $\mathbf{1 8 . 7}(\boldsymbol{n}=\mathbf{3 1})$ & $\mathbf{0 . 0 2}$ \\
AVK & $8.2(n=9)$ & $14.3(n=8)$ & $10.2(n=17)$ & 0.22 \\
Immunosuppresor & $4.5(n=5)$ & $5.4(n=3)$ & $4.8(n=8)$ & 0.82 \\
Femoral hernia & $28.2(n=31)$ & $25(n=14)$ & $27.1(n=45)$ & 0.66 \\
Inguinal hernia & $50(n=55)$ & $55.4(n=31)$ & $51.8(n=86)$ & 0.51 \\
Umbilical hernia & $21.8(n=24)$ & $23.2(n=13)$ & $22.3(n=37)$ & 0.84 \\
Omentum & $36.1(n=39)$ & $28.6(n=16)$ & $33.5(n=55)$ & 0.33 \\
Colon & $10.2(n=11)$ & $17.9(n=10)$ & $12.8(n=21)$ & 0.16 \\
Small bowel & $48.1(n=52)$ & $61.8(n=34)$ & $52.8(n=86)$ & 0.1 \\
Congestive bowel & $41.4(n=36)$ & $25.5(n=13)$ & $35.5(n=49)$ & 0.06 \\
Clear liquid & $30(n=21)$ & $23.5(n=8)$ & $27.9(n=29)$ & 0.49 \\
Turbid liquid & $14.3(n=10)$ & $117.6(n=6)$ & $15.4(n=16)$ & 0.66 \\
Mesh & $38.2(n=42)$ & $39.3(n=22)$ & $37.5(n=64)$ & 0.89 \\
Resection & $\mathbf{7 . 3 ( n = 8 )}$ & $\mathbf{3 0 . 4 ( n = 1 7 )}$ & $\mathbf{1 5 . 1}(\boldsymbol{n}=\mathbf{2 5})$ & $<\mathbf{0 . 0 0 1}$ \\
\hline & & & &
\end{tabular}

even patients with concomitant bowel resection can safely be managed by use of mesh.

Surgical characteristics and outcome of our cohort are in accordance with the literature. A recent study reported that strangulated groin hernias represented around $1.7 \%$ of all hernia repairs; hernia sites were inguinal in $61 \%$ and femoral in $39 \%$ of patients [24]. Morbidity and mortality after surgery for incarcerated
Table 5 Univariate risk factors for major complications
Entries in bold inside the table are stastistically significant results

\begin{tabular}{lllll}
\hline & No Clavien $3 / 5, \%(\mathrm{n}=144)$ & Clavien 3/5, \% $(n=22)$ & Total, \% $(n=166)$ & $P$ value \\
\hline Female & $39.6(n=57)$ & $54.5(n=12)$ & $41.6(n=69)$ & 0.18 \\
ASA $>2$ & $34.7(n=26)$ & $53.8(n=7)$ & $37.5(n=33)$ & 0.29 \\
Cardiopathy & $29.2(n=42)$ & $45.5(n=10)$ & $31.3(n=52)$ & 0.12 \\
BPCO & $14.6(n=21)$ & $9.1(n=2)$ & $13.9(n=23)$ & 0.49 \\
Diabetes & $14.6(n=21)$ & $18.2(n=4)$ & $15.1(n=25)$ & 0.66 \\
Obesity & $17.4(n=25)$ & $4.5(n=1)$ & $15.7(n=26)$ & 0.12 \\
Aspirin & $18.8(n=27)$ & $18.2(n=4)$ & $18.7(n=31)$ & 0.95 \\
AVK & $10.4(n=15)$ & $9.1(n=2)$ & $10.2(n=17)$ & 0.85 \\
Immunosuppressor & $4.9(n=7)$ & $4.5(n=1)$ & $4.8(n=8)$ & 0.95 \\
Femoral hernia & $\mathbf{2 4 . 3 ( n = 3 5 )}$ & $\mathbf{4 5 . 5}(\boldsymbol{n}=\mathbf{1 0})$ & $\mathbf{2 7 . 1}(\boldsymbol{n}=\mathbf{4 5})$ & $\mathbf{0 . 0 4}$ \\
Inguinal hernia & $52.8(n=76)$ & $45.5(n=10)$ & $51.8(n=86)$ & 0.52 \\
Umbilical hernia & $22.9(n=33)$ & $18.2(n=4)$ & $22.3(n=37)$ & 0.62 \\
Omentum & $35.2(n=50)$ & $22.7(n=5)$ & $33.5(n=55)$ & 0.25 \\
Colon & $12.7(n=18)$ & $13.6(n=3)$ & $12.8(n=21)$ & 0.9 \\
Small bowel & $\mathbf{4 9 . 6 ( n = 7 0 )}$ & $\mathbf{7 2 . 7 ( n = 1 6 )}$ & $\mathbf{5 2 . 8}(\boldsymbol{n}=\mathbf{8 6})$ & $\mathbf{0 . 0 4}$ \\
Congestive bowel & $36.2(n=42)$ & $31.8(n=7)$ & $35.5(n=49)$ & 0.69 \\
Clear liquid & $28(n=26)$ & $27.3(n=3)$ & $27.9(n=29)$ & 0.96 \\
Turbid liquid & $14(n=13)$ & $27.3(n=3)$ & $15.4(n=16)$ & 0.25 \\
Mesh & $38.2(n=55)$ & $40.9(n=9)$ & $38.6(n=64)$ & 0.81 \\
Resection & $\mathbf{1 1 . 8 ( n = 1 7 )}$ & $\mathbf{3 6 . 4 ( n = 8 )}$ & $\mathbf{1 5 . 1}(\boldsymbol{n}=\mathbf{2 5})$ & $\mathbf{0 . 0 0 3}$ \\
\hline & & & &
\end{tabular}


Table 6 Univariate risk factors for postoperative SSI
Entries in bold inside the table are stastistically significant results

\begin{tabular}{lllll}
\hline & $\begin{array}{l}\text { No surgical site, } \% \\
\text { infection }(n=158)\end{array}$ & $\begin{array}{l}\text { Surgical site infection, } \\
\%(n=8)\end{array}$ & Total $(n=166)$ & $P$ value \\
\hline Female & $41.8(n=66)$ & $37.5(n=3)$ & $41.6(n=69)$ & 0.81 \\
Cardiopathy & $29.7(n=47)$ & $62.5(n=5)$ & $31.3(n=52)$ & 0.051 \\
BPCO & $13.9(n=22)$ & $12.5(n=1)$ & $13.9(n=23)$ & 0.91 \\
Diabetes & $14.6(n=23)$ & $25(n=2)$ & $15.1(n=25)$ & 0.42 \\
ASA $>2$ & $39.3(n=33)$ & 0 & $37.5(n=33)$ & 0.11 \\
Obesity & $15.2(n=24)$ & $25(n=2)$ & $15.7(n=26)$ & 0.45 \\
Aspirin & $19(n=30)$ & $12.5(n=1)$ & $18.7(n=31)$ & 0.65 \\
AVK & $9.5(n=15)$ & $25(n=2)$ & $10.2(n=17)$ & 0.16 \\
Immunosuppressor & $5.1(n=8)$ & 0 & $4.8(n=8)$ & 0.51 \\
Femoral hernia & $27.2(n=43)$ & $25(n=2)$ & $27.1(n=45)$ & 0.89 \\
Inguinal hernia & $52.5(n=83)$ & $37.5(n=3)$ & $51.8(n=86)$ & 0.4 \\
Umbilical hernia & $20.9(n=33)$ & $50(n=4)$ & $22.3(n=37)$ & 0.054 \\
Omentum & $33.3(n=52)$ & $37.5(n=3)$ & $33.5(n=55)$ & 0.8 \\
Colon & $\mathbf{1 1 . 5}(n=\mathbf{1 8})$ & $\mathbf{3 7 . 5 ( n = 3 )}$ & $\mathbf{1 2 . 8}(\boldsymbol{n}=\mathbf{2 1})$ & $\mathbf{0 . 0 3}$ \\
Small bowel & $52.9(n=82)$ & $50(n=4)$ & $52.8(n=86)$ & 0.87 \\
Congestive bowel & $35.4(n=46)$ & $37.5(n=3)$ & $35.5(n=49)$ & 0.9 \\
Turbid liquid & $15.3(n=15)$ & $16.7(n=1)$ & $15.4(n=16)$ & 0.9 \\
Mesh & $38.6(n=61)$ & $37.5(n=3)$ & $38.6(n=64)$ & 0.95 \\
Resection & $14.6(n=23)$ & $25(n=2)$ & $15.1(n=25)$ & 0.42 \\
\hline
\end{tabular}

hernia were reported to be as high as 21-39 and 4-5\%, respectively [24-28].

In our work, five factors were associated with overall complications: bowel resection, treatment by aspirin, medical history of diabetes, cardiopathy, and ASA score $>2$. Risk factors for morbidity are various according to studies. The factor reported as the sole factor affecting morbidity and mortality was intestinal necrosis followed by bowel resection [25].

The other factors have not been reported in the literature, but it can be easy to explain the role of aspirin and cardiopathy (often treated by antiaggregant) in the formation of hematoma or in general complication.

Moreover, a recent study reported that first intention exploratory laparotomy was a major cause of postoperative complication [24]. This was not tested in our work, but authors concluded that the best way for exploring abdominal cavity was hernioscopy or laparoscopy [24].

Table 7 Multivariate analysis risk factor for overall morbidity

\begin{tabular}{llll}
\hline Overall morbidity & Odds ratio & $95 \%$ Confidence interval & $P$ value \\
\hline Mesh & 1.515 & $0.51-4.53$ & 0.458 \\
Femoral hernia & 1 & - & - \\
Inguinal hernia & 1.534 & $0.47-5$ & 0.478 \\
Umbilical hernia & 0.799 & $0.16-4$ & 0.786 \\
Bowel incarceration & 3.225 & $1.13-9.18$ & 0.028 \\
ASA score $>2$ & 2.912 & $1.05-8.1$ & 0.041 \\
\hline
\end{tabular}

Risk factors for severe complications in our study were intestinal resection and femoral hernia site which is probably directly correlated with the first one, as incarcerated hernias require more frequent bowel resections (as we can see in our work). This factor was confirmed by multivariate analysis. Interestingly, none of the assessed patient-related risk factors was associated with major complications.

ASA score is not associated with the risk of major complication but with the risk of overall morbidity. This difference could be explained by the fact that ASA score reflects the status of the patient as a whole. Complications related to ASA score are more often medical complications that could be treated without anesthesia or intensive care.

The only significant risk factor for SSI was incarceration of the colon that has not been previously reported in the literature. However, colorectal procedures are associated with high SSI rates between 5.2 and 8.9\% [29]. Of note, Ueda et al. [16] reported $20 \%$ SSI in their overall population with no

Table 8 Multivariate analysis risk factor for major complication

\begin{tabular}{llll}
\hline Major complications & Odds ratio & $95 \%$ Confidence interval & $P$ value \\
\hline Mesh & 1.21 & $0.32-4.59$ & 0.77 \\
Femoral hernia & 1 & - & - \\
Inguinal hernia & 0.58 & $0.14-2.39$ & 0.46 \\
Umbilical hernia & 0.56 & $0.08-4.11$ & 0.57 \\
Bowel incarceration & 14.04 & $1.71-115.2$ & 0.01 \\
ASA score $>2$ & 1.58 & $0.44-5.65$ & 0.48 \\
\hline
\end{tabular}


significant difference between the group mesh and no-mesh. A more recent study was more reassuring with a rate of mesh infection of $1.25 \%$ and a rate of wound infection of $11.25 \%$ [27]. Several studies conclude that the use of mesh does not increase the risk for complication in the absence of bowel resection [4, 11, 27, 30-32]. When bowel resection is needed, some studies suggest that mesh repair could be used [16, 27]. Bessa et al reported $5.6 \%$ of SSI in case of bowel resection, and Ueda et al $18 \%[16,27]$. In the last study, no infection of prosthesis occurred and no mesh has been removed. In our study, the observed SSI rate was $3.9 \%$ with mesh repair, and no single infection required mesh removal in our patients.

The reason to use prosthesis despite strangulation in hernia repairs is the significant reduced hernia recurrence from 11.1 and $33.3 \%$ in suture to 0 and $4.7 \%$ with mesh $[11,30]$. In case of paraumbilical hernia, the same observations have been made, and after a follow-up of 16 months, use of prosthetic repair led to superior results in terms of recurrence without increase of morbidity [33]. However, these data from elective surgery series have yet to be confirmed for emergency surgeries for incarcerated hernias. Lohsiriwat et al. published a series of long term follow-up of hernia repair proceeded in emergency [14]. They reported a ratio of recurrence of $10 \%$ for Lichtenstein repair within 2.5 to 7.6 years.

This present study has several limitations inherent to its retrospective nature. Some data were missing, and complications might be underestimated. Further, surgical treatment was heterogeneous and decided by the surgeon on call. However, it was exactly the intent to report current practice in our institutions, and comprehensive guidelines for the use of mesh do not exist so far. Our study cohort is limited, and number of infections was too low to perform specific multivariate analysis. The risk factors identified by univariate analysis are likely to be confounded and have therefore to be interpreted with caution. We cannot provide long-term follow-up. Therefore, we cannot prove superiority of mesh repairs with regard to potentially lower recurrence rates. Nonetheless, we provide a welldocumented audit of our institutional practice that contributes to the body of evidence in a controversial field of surgery where high-level evidence is scarce and difficult to obtain.

\section{Conclusion}

In conclusion, our study confirmed high morbidity after emergency surgery for incarcerated hernia. Mesh repair could be used and appears to be safe. Some elements are in favor of the use and the safety of prosthesis even in case of concomitant bowel resection. Larger prospective cohorts are needed to confirm our findings.

\section{Conflicts of interest None.}

\section{References}

1. Gallegos NC, Dawson J, Jarvis M, Hobsley M (1991) Risk of strangulation in groin hernias. Br J Surg 78:1171-1173

2. Kulah B, Kulacoglu IH, Oruc MT, Duzgun AP, Moran M, Ozmen MM, Coskun F (2001) Presentation and outcome of incarcerated external hernias in adults. Am J Surg 181:101-104

3. Kurt N, Oncel M, Ozkan Z, Bingul S (2003) Risk and outcome of bowel resection in patients with incarcerated groin hernias: retrospective study. World J Surg 27:741-743

4. Derici H, Unalp HR, Nazli O, Kamer E, Coskun M, Tansug T, Bozdag AD (2010) Prosthetic repair of incarcerated inguinal hernias: is it a reliable method? Langenbecks Arch Surg 395:575-579

5. Mathonnet M, Mehinto D (2007) Anterolateral hernias of the abdomen. J Chir 144(4):5S19-5S22

6. Jenkins JT, O’Dwyer PJ (2008) Inguinal hernia. BMJ 336:268-272

7. Danielsson P, Isacson S, Hansen MV (1999) Randomised study of Lichtenstein compared with Shouldice inguinal hernia repair by surgeons in training. Eur J Surg 165:49-53

8. McGillicuddy JE (1998) Prospective randomized comparison of the Shouldice and Lichtenstein hernia repair procedures. Arch Surg 133: 974-978

9. Butters M, Redecke J, Köninger J (2007) Long-term results of a randomized clinical trial of Shouldice, Lichtenstein and transabdominal preperitoneal hernia repairs. Br J Surg 94:562-565

10. Bisgaard T, Bay-Nielsen M, Christensen IJ, Kehlet H (2007) Risk of recurrence 5 years or more after primary Lichtenstein mesh and sutured inguinal hernia repair. Br J Surg 94:1038-1040

11. Elsebae MMA, Nasr M, Said M (2008) Tension free repair versus Bassini technique for strangulated inguinal hernia: a controlled randomized study. Int J Surg 6:302-305

12. Pans A, Desive C, Jacquet N (1997) Use of a preperitoneal prosthesis for strangulated groin hernia. Br J Surg 84:310-312

13. Lohsiriwat V, Sridermma W, Akaraviputh T, Boonnuch W, Chinsawangwatthanakol V, Methasate A et al (2007) Surgical outcomes of Lichtenstein tension-free hernioplasty for acutely incarcerated hernia. Surg Today 37:212-214

14. Lohsiriwat D, Lohsiriwat S (2013) Long-term outcomes of emergency Lichtenstein hernioplasty for incarcerated inguinal hernia. Surg Today 43:990-994

15. Atila K, Guler S, Inal A, Sokmen S, Karademir S, Bora S (2010) Prosthetic repair of acutely incarcerated groin hernias: a prospective clinical observational cohort study. Langenbecks Arch Surg 395: $563-568$

16. Ueda J, Nomura T, Sasaki et al (2012) Prosthetic repair of an incarcerated groin hernia with small intestinal resection. Surg Today 42:359-362

17. Garner JS (1986) CDC guideline for prevention of surgical wound infections, 1985. Supercedes guideline for prevention of surgical wound infection published in 1982. Revised Infect Control 7:193200

18. Simmons BP (1982) Guideline for prevention of surgical wound infections. Infect Control 3:185-196

19. Mangram AJ, Horan TC, Pearson ML, Silver LC, Jarvis WR (1999) Guideline for prevention of surgical site infection, 1999. Center for Disease Control and Prevention (CDC) Hospital Infection Control Practices Advisory Committee. Am J Infect Control 27:97-132

20. Pélissier E, Ngo P (2007) Traitement des hernies de l'aine étranglées. EMC. Elsevier Masson SAS, Paris, Techniques chirurgicalesAppareil digestif, 40-139

21. Expert Panel on the Identification, Evaluation and Treatment of Overweight in adult (1998) Clinical guidelines on the identification, evaluation and treatment of overweight and obesity in adults: executive summary: expert panel on the identification, evaluation and treatment of overweight in adults. Am J Clin Nutr 68:899-917 
22. Dindo D, Demartines N, Clavien PA (2004) Classification of surgical complications. A new proposal with evaluation in a cohort of 6336 patients and results of a survey. Ann Surg 244:931-937

23. Horan TC, Gaynes RP, Martone WJ, Jarvis WR, Emori TG (1992) CDC definitions of nosocomial surgical site infection, 1992: a modification of CDC definitions of surgical wound infections. Infect Control Hosp Epidemiol 13:606-608

24. Romain B, Chemaly R, Meyer N, Brigand C, Steinmetz JP, Rohr S (2012) Prognostic factors of postoperative morbidity and mortality in strangulated groin hernia. Hernia 16:405-410

25. Derici H, Unalp HR, Bozdag AD, Nazli TT, Kamer E (2007) Factors affecting morbidity and mortality in abdominal wall hernias. Hernia 11:341-346

26. Martinez-Serrano MA, Pereira JA, Sancho J, Argudo N, Lopez-Cano M, Grande L (2012) Specific improvement measures to reduce complications and mortality after urgent surgery in complicated abdominal wall hernia. Hernia 16:171-177

27. Bessa SS, Abdel-Razek AH (2013) Results of prosthetic mesh repair in the emergency management of the acutely or strangulated ventral hernias: a seven years study. Hernia 17:59-65

28. Matinez-Serrano MA, Pereira JA, Sancho JJ, Lopez-Cano J, Bombuy E, Hidlago $J$ et al (2010) Risk of death after emergency repair of abdominal wall hernias. Still waiting for improvement. Langenbecks Arch Surg 395:551-556

29. Kwaan MR, Al-Refaie WB, Parsons HM, Chow CJ, Rothenberger DA, Habermann EB (2013) Are right-sided colectomy outcomes different from left-sided colectomy outcomes?: study of patients with colon cancer in the ACS NSQIP database. JAMA Surg 148:504-510

30. Papaziogas B, Lazaridis C, Makris J, Koutelidakis J, Patsas A, Grigoriou M, Chatzimavroudis G, Psaralexis K, Atmatzidis K (2005) Tension-free repair versus modified Bassini technique (Andrews technique) for strangulated inguinal hernia: a comparative study. Hernia 9:156-159

31. Wysocki A, Kulawik J, Pozniczek M, Strzalka M (2006) Is the Lichtenstein operation of strangulated groin hernia a safe procedure? World J Surg 30:2065-2070

32. Bessa SS, Katri KM, Abdel-Salam WN, Abdel-Baki NA (2007) Early results from the use of the Liechtenstein repair in the management of strangulated groin hernia. Hernia 11: 239-242

33. Abdel-Baki NA, Bessa SS, Abdel-Razek AH (2007) Comparison of prosthetic mesh repair and tissue repair in the emergency management of incarcerated para-umbilical hernia: a prospective randomized study. Hernia 11:163-167 